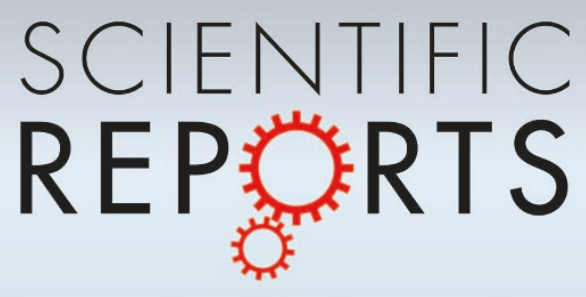

OPEN

SUBJECT AREAS:

RAMAN SPECTROSCOPY

SURFACE SPECTROSCOPY

SCANNING PROBE

MICROSCOPY

OPTICAL PROPERTIES AND

DEVICES

Received

3 July 2013

Accepted

3 September 2013

Published

19 September 2013

Correspondence and requests for materials should be addressed to S.R. (sunryu@khu.ac.

$\mathrm{kr})$

* These authors contributed equally to this work.

\section{Origin of New Broad Raman D and G Peaks in Annealed Graphene}

\author{
Jinpyo Hong*, Min Kyu Park*, Eun Jung Lee*, DaeEung Lee, Dong Seok Hwang \& Sunmin Ryu
}

Department of Applied Chemistry, Kyung Hee University, 1 Seocheon, Yongin, Gyeonggi 446-701, Korea.

Since graphene, a single sheet of graphite, has all of its carbon atoms on the surface, its property is very sensitive to materials contacting the surface. Herein, we report novel Raman peaks observed in annealed graphene and elucidate their chemical origins by Raman spectroscopy and atomic force microscopy (AFM). Graphene annealed in oxygen-free atmosphere revealed very broad additional Raman peaks overlapping the $D, G$ and 2D peaks of graphene itself. Based on the topographic confirmation by AFM, the new Raman peaks were attributed to amorphous carbon formed on the surface of graphene by carbonization of environmental hydrocarbons. While the carbonaceous layers were formed for a wide range of annealing temperature and time, they could be effectively removed by prolonged annealing in vacuum. This study underlines that spectral features of graphene and presumably other 2-dimensional materials are highly vulnerable to interference by foreign materials of molecular thickness.

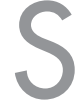

ince its first mechanical isolation from graphite in $2004^{1}$, graphene has been a subject of more than 20,000 research papers revealing a variety of its extraordinary materials properties. Such a drastic expansion has been boosted not only by various simple methods for exfoliation ${ }^{1,2}$ and synthesis ${ }^{3}$, but also by the stability of the resulting graphene samples under various external perturbations $s^{4}$. Like graphite, graphene is chemically inert that treatments with most chemicals cause no structural damage and functionalization at the basal plane requires either mineral acids in the presence of strong oxidants ${ }^{2}$ or radical species ${ }^{5,6}$. Graphene also remains intact at elevated temperatures in oxygen-free atmosphere ${ }^{7}$. The thermal stability, in particular, has allowed heating graphene up to several hundred degree $\mathrm{C}$ without severe degradation in preparing various types of graphene devices $^{1}$, cleaning surfaces for scanning probe microscopy ${ }^{8,9}$ and molecular assembly ${ }^{10}$, and chemical vapor deposition (CVD) of hexagonal BN with graphene as substrates ${ }^{11}$.

Despite the apparent thermal stability, however, thermal treatments cause graphene non-negligible changes in its material properties. Graphene supported on $\mathrm{SiO}_{2} / \mathrm{Si}$ substrates, for example, undergoes in-plane compression $^{12,13}$ and rippling ${ }^{14}$ due to conformal adhesion ${ }^{15}$ or differential thermal expansion ${ }^{13,16}$ of graphene with respect to the substrates upon annealing at $>100^{\circ} \mathrm{C}$. At the same time with the compression, annealed graphene becomes strongly p-doped by the ambient oxygen molecules ${ }^{14}$. As a result of both structural and electronic modifications, the charge carrier mobility of annealed graphene devices is much lower than that of pristine devices ${ }^{17}$. For graphene samples prepared by the mechanical exfoliation method using adhesive tape, heating is likely to induce contamination of graphene with nearby polymeric adhesive residues which may become fluidic or airborne when thermally decomposed ${ }^{7}$. Similar phenomenon may occur in CVD-grown graphene transferred onto solid substrates using polymeric supports which cannot be removed completely by solvent cleaning ${ }^{10}$. Notwithstanding its practical importance in understanding and improving properties of graphene sheets and devices, the annealinginduced contamination of graphene surface has not been investigated in a systematic manner.

Herein we report that novel broad Raman peaks emerge when graphene is annealed in oxygen-free environment and reveal that they originate not from graphene itself but from amorphous carbon $(\mathrm{aC})$ generated from residual hydrocarbons at elevated temperature. This study also provides a straightforward optical method to monitor the surface quality of graphene, bearing practical importance towards many related fields.

\section{Results}

Figure $1 \mathrm{a}$ and $1 \mathrm{~b}$ show the optical micrographs of exfoliated single layer graphene (denoted by $1 \mathrm{~L}$, spanning $\sim 17$ $\left.\times 14 \mu \mathrm{m}^{2}\right)$, obtained before and after thermal annealing in Ar atmosphere $\left(\mathrm{T}_{\text {anneal }}=400^{\circ} \mathrm{C}\right.$; $\tau_{\text {anneal }}=2 \mathrm{hrs}$ ). (See experimental section for detailed methods.) Whereas graphene and thick graphite in yellow color remain optically identical after the annealing, adhesive residue marked by the yellow arrow disappeared through thermal decomposition of the polymeric materials. The Raman spectrum obtained from graphene in its pristine state (red 
a

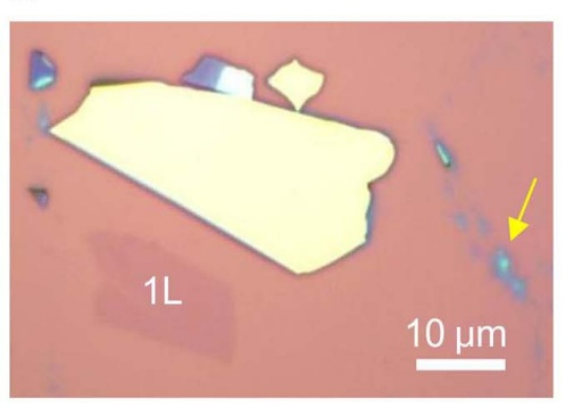

b

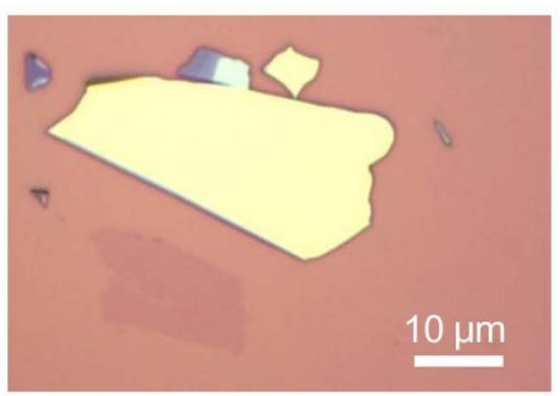

C

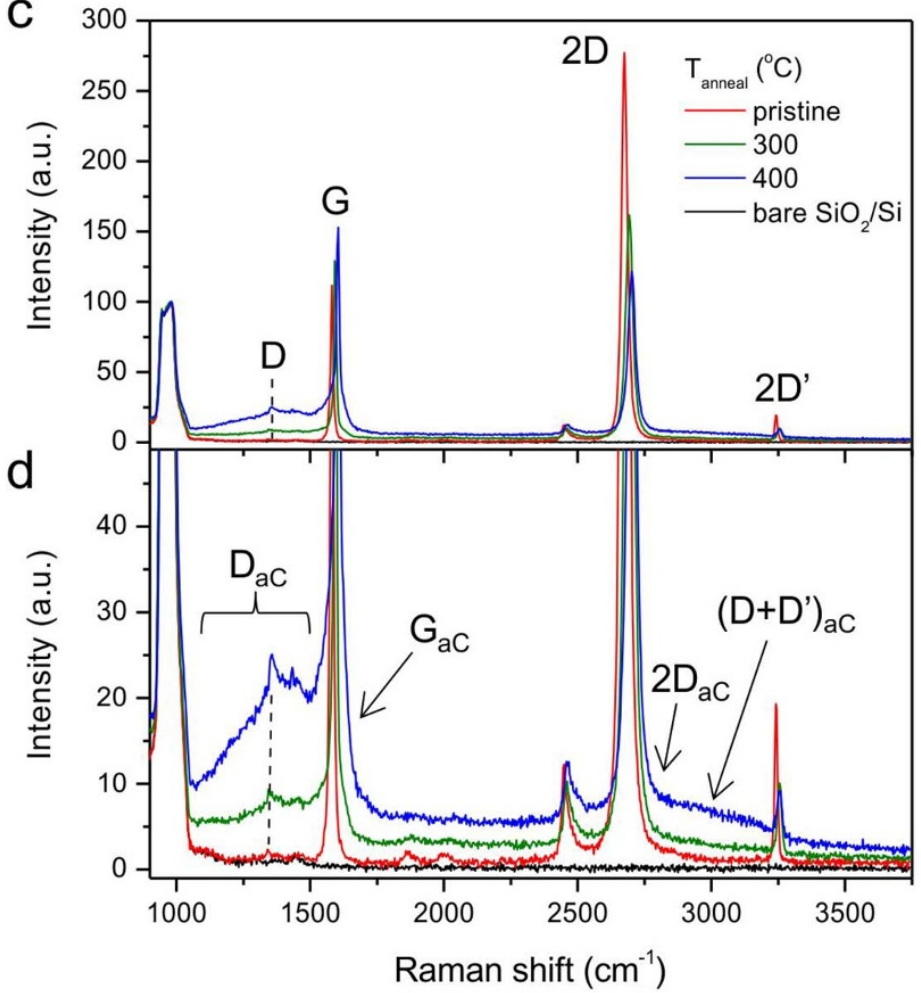

Figure $1 \mid$ The effects of thermal annealing in single layer (1L) graphene. (a), (b) Optical micrograph of pristine and annealed graphene/SiO ${ }_{2} / \mathrm{Si}$, respectively $\left(\mathrm{T}_{\text {anneal }}=400^{\circ} \mathrm{C} ; \tau_{\text {anneal }}=30 \mathrm{~min}\right.$; in Ar gas). The yellow arrow points to polymeric adhesive residues. (c) Raman spectra of pristine (red) and annealed graphene with that of bare substrates (black). (d) Expanded view of (c).

in Fig. 1c) shows only one first-order Raman peak at $\sim 1580 \mathrm{~cm}^{-1}$, denoted by $\mathrm{G}$, which originates from the doubly degenerate zonecenter phonon $\mathrm{E}_{2 \mathrm{~g}}$ mode and corresponds to the $\mathrm{C}$ - $\mathrm{C}$ stretching mode ${ }^{18}$. The D peak $\left(\sim 1340 \mathrm{~cm}^{-1}\right)$ arising from the TO phonon mode near $\mathrm{K}$ points in the Brillouin zone is hardly detectible in the pristine graphene. Since it is only activated by structural defects by a second order Raman scattering process through the intervalley double resonance, lack of the peak represents high crystallinity of the sample $^{18}$. Graphene with significant defects would show an additional disorder related peaks, $\mathrm{D}^{\prime}$ and $\left(\mathrm{D}+\mathrm{D}^{\prime}\right)$ at $\sim 1620$ and $\sim 2950 \mathrm{~cm}^{-1}$, originating from the intravalley double resonance and a combination mode, respectively ${ }^{5,6}$. The second order Raman scattering in the pristine graphene also generated $2 \mathrm{D}$ and $2 \mathrm{D}^{\prime}$ peaks at 2680 and $3240 \mathrm{~cm}^{-1}$, respectively, overtones of $\mathrm{D}$ and $\mathrm{D}^{\prime}$ modes ${ }^{19}$. Most of the pristine samples including the one in Fig. 1 showed D/G peak height ratio $\left(\mathrm{I}_{\mathrm{D}} / \mathrm{I}_{\mathrm{G}}\right)$ of $\sim 1 \%$ confirming high crystallinity ${ }^{7}$, while few with unusually high values were discarded to avoid complication.

When annealed for 2 hours at $300^{\circ} \mathrm{C}$ in Ar gas, the spectrum shows decrease in 2D's intensity and upshifts in the frequencies of $G$ and $2 \mathrm{D}$, both of which are mostly due to annealing-induced hole doping caused by ambient $\mathrm{O}_{2}$ molecules ${ }^{13,14}$ with minor contribution from lattice compression ${ }^{13}$. We also note that the spectrum background between 1100 and $1600 \mathrm{~cm}^{-1}$ increased noticeably. The spectral changes are more obvious in the expanded viewgraph in Fig. 1d. For the sample annealed at $400^{\circ} \mathrm{C}$, for example, the broad peak is clearly seen to be centered near the D peak. A close look further reveals that broad features also exist around $\mathrm{G}$ and $2 \mathrm{D}$ peaks, respectively. In particular, the spectrum near $1600 \mathrm{~cm}^{-1}$ can be decomposed into a sharp $\mathrm{G}$ and a broad peak, located at 1603 and $1587 \mathrm{~cm}^{-1}$, respectively (see Supplementary Fig. S1 online). The broad Raman peaks near $\mathrm{D}, \mathrm{G}$ and $2 \mathrm{D}$ regions are strongly reminiscent of $s p^{2}$-rich amorphous carbons ${ }^{20,21}$. Indeed, the broad spectral features match well with the spectrum obtained from commercial carbon black powder, a form of amorphous carbons (see Supplementary Fig. S2). Thus we attribute the annealing-induced broad Raman peaks, denoted by $\mathrm{D}_{\mathrm{aC}}, \mathrm{G}_{\mathrm{aC}}, 2 \mathrm{D}_{\mathrm{aC}}$ and $\left(\mathrm{D}+\mathrm{D}^{\prime}\right)_{\mathrm{aC}}$ to amorphous carbons $(\mathrm{aC})$ deposited on graphene through carbonization ${ }^{22}$ of hydrocarbons during the thermal treatments in inert atmosphere. Since the broad Raman peaks were not observed on the bare substrates near graphene flakes as explained below, it can be concluded that the adsorption of hydrocarbons and their carbonization occur selectively on the surface of graphene. The hydrocarbons, carbon source of aC, are thought to be the adhesive polymers left on the substrates during the mechanical exfoliation of graphene, since the polymeric residues were apparently removed by annealing and no other carbon source was available. We also found that the aC-derived Raman peaks occur in CVD-grown graphene transferred onto $\mathrm{SiO}_{2} / \mathrm{Si}$ substrates using poly(methyl methacrylate) (PMMA) supports ${ }^{23}$. Since removal of PMMA by acetone is known to be incomplete ${ }^{10,24}$, these peaks are attributed to PMMA residues on graphene. (See Supplementary Figs. S3 and S4 online for detailed spectra and ensuing discussion.)

In Fig. 2a, we investigated the effects of the gas environment in the furnace quartz tube on the intensity of $\mathrm{D}_{\mathrm{aC}}, \mathrm{I}\left(\mathrm{D}_{\mathrm{aC}}\right)$, of exfoliated graphene. Since $\mathrm{D}_{\mathrm{aC}}$ cannot be separated unambiguously from the rest Raman features, $\mathrm{I}\left(\mathrm{D}_{\mathrm{aC}}\right)$ was arbitrarily defined as the intensity at $1280 \mathrm{~cm}^{-1}$ to avoid overlap with the D peak from graphene itself. For quantitative comparison, all the spectra were normalized by the intensity of the Raman peak located at $\sim 960 \mathrm{~cm}^{-1}$ which originates from underlying Si substrates. Whereas $I\left(D_{a C}\right)$ reached $\sim 15 \%$ of the Si's reference peak when annealed in inert Ar atmosphere or reducing forming gas $\left(5 \% \mathrm{H}_{2} / 95 \% \mathrm{~N}_{2}\right)$ at $400^{\circ} \mathrm{C}$, it decreased down to $\sim 8 \%$ in a low vacuum $\left(\sim 2 \times 10^{-3}\right.$ Torr $)$. As decreasing the base pressure to a high vacuum level, $\mathrm{I}\left(\mathrm{D}_{\mathrm{aC}}\right)$ was further attenuated. This suggests that gaseous hydrocarbons emitted from the polymers at elevated temperature are lingering in the reaction chamber and then adsorb on graphene followed by carbonization to form aC.

The duration of annealing $\left(\tau_{\text {anneal }}\right)$ was also found to affect $I\left(D_{a C}\right)$ as shown in Fig. 2 b. $I\left(D_{a C}\right)$ peaked at the smallest $\tau_{\text {anneal }}$ of 0.5 hour in 


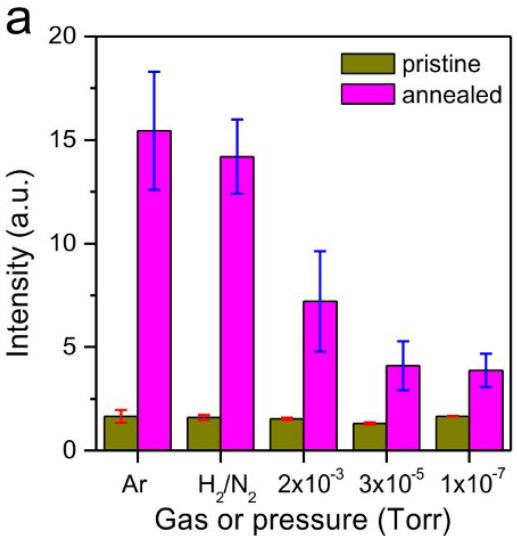

b

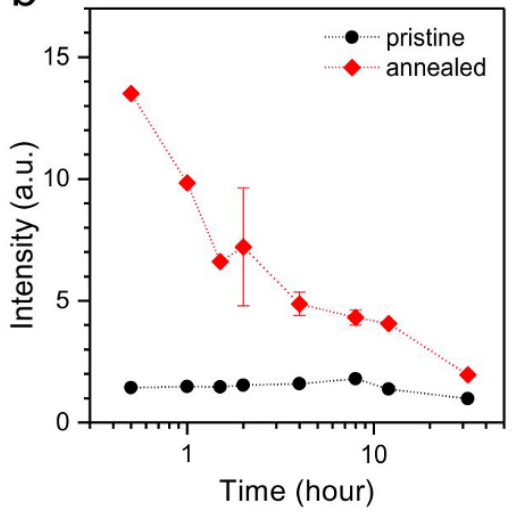

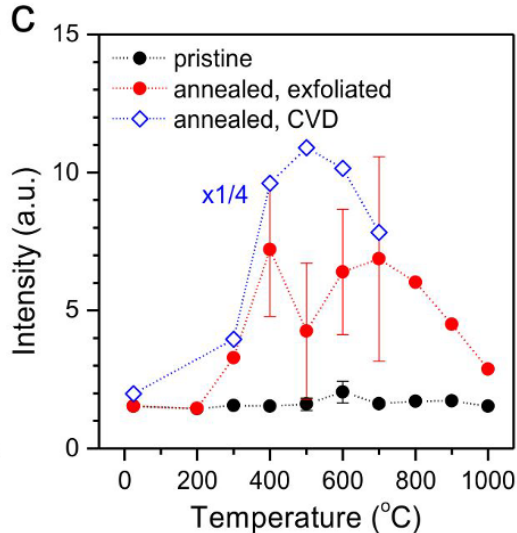

Figure $2 \mid$ The effects of various environmental parameters on the intensity of $D_{a c}$ peak, $I\left(D_{a C}\right)$. (a) $I\left(D_{a C}\right)$ of graphene obtained after annealing in various gas environment of the tube furnace $\left(\mathrm{T}_{\text {anneal }}=400^{\circ} \mathrm{C}, \tau_{\text {anneal }}=2\right.$ hours $)$. The first two measurements were carried out in Ar and forming gas of $\sim 760$ Torr, respectively, while the rest were in vacuum of the specified base pressure. $I\left(D_{a C}\right)$ of each pristine sample is shown together for comparison. (b) $\mathrm{I}\left(\mathrm{D}_{\mathrm{aC}}\right)$ of graphene for varying $\tau_{\text {anneal }}\left(\mathrm{T}_{\text {anneal }}=400^{\circ} \mathrm{C}\right.$, in low vacuum). (c) $\mathrm{I}\left(\mathrm{D}_{\mathrm{aC}}\right)$ of exfoliated and CVD-grown graphene for varying $\mathrm{T}_{\text {anneal }}\left(\tau_{\text {anneal }}=2\right.$ hours). The exfoliated graphene was annealed in low vacuum, while the CVD-grown graphene was in Ar gas. Each of data point in (a) $\sim$ (c) represents a statistical average for $20 \sim 30$ separate spectra from more than 3 graphene samples.

a low vacuum and decreased to a half for $\tau_{\text {anneal }}=2$ hours. Extended thermal treatment led to almost zero intensity of $\mathrm{D}_{\mathrm{aC}}$, suggesting that the surface of graphene is almost free of aC. Note that nonzero values for pristine graphene, $\mathrm{I}\left(\mathrm{D}_{\mathrm{aC}}\right) \sim 1.5 \%$, is mostly due to unidentified optical scattering from the underlying substrates, not graphene or $\mathrm{aC}$ as can be seen in Fig. 1d. When $\mathrm{T}_{\text {anneal }}$ was varied for exfoliated graphene, $\mathrm{I}\left(\mathrm{D}_{\mathrm{aC}}\right)$ started to increase at $300^{\circ} \mathrm{C}$ and reached $7.2 \% \pm$ $2.4 \%$ at $400^{\circ} \mathrm{C}$ as shown in Fig. 2c. At even higher temperature $\left(>800^{\circ} \mathrm{C}\right)$, however, $\mathrm{I}\left(\mathrm{D}_{\mathrm{aC}}\right)$ decreased with increasing $\mathrm{T}_{\text {anneal }}$. Rather large error bars in $\mathrm{I}\left(\mathrm{D}_{\mathrm{aC}}\right)$ are due to sample-to-sample variation, which possibly originates from random distribution of adhesive residues around graphene flakes of interest. A similar $\mathrm{T}_{\text {anneal }}$-dependence was observed for CVD-grown graphene but with $\mathrm{I}\left(\mathrm{D}_{\mathrm{aC}}\right)$ overall higher than that for exfoliated graphene.

Using AFM, we were able to visualize overlayers of aC on graphene formed in $\mathrm{Ar}$ atmosphere at $700^{\circ} \mathrm{C}$. For this characterization, bilayer graphene (2L) shown in Fig. 3a was employed since 1L graphene is severely etched by a carbothermal reduction of underlying $\mathrm{SiO}_{2}{ }^{25}$. To expose $\mathrm{SiO}_{2}$ surface within the graphene basal plane as a height reference, etch pits were generated by oxidizing ${ }^{7}$ graphene at
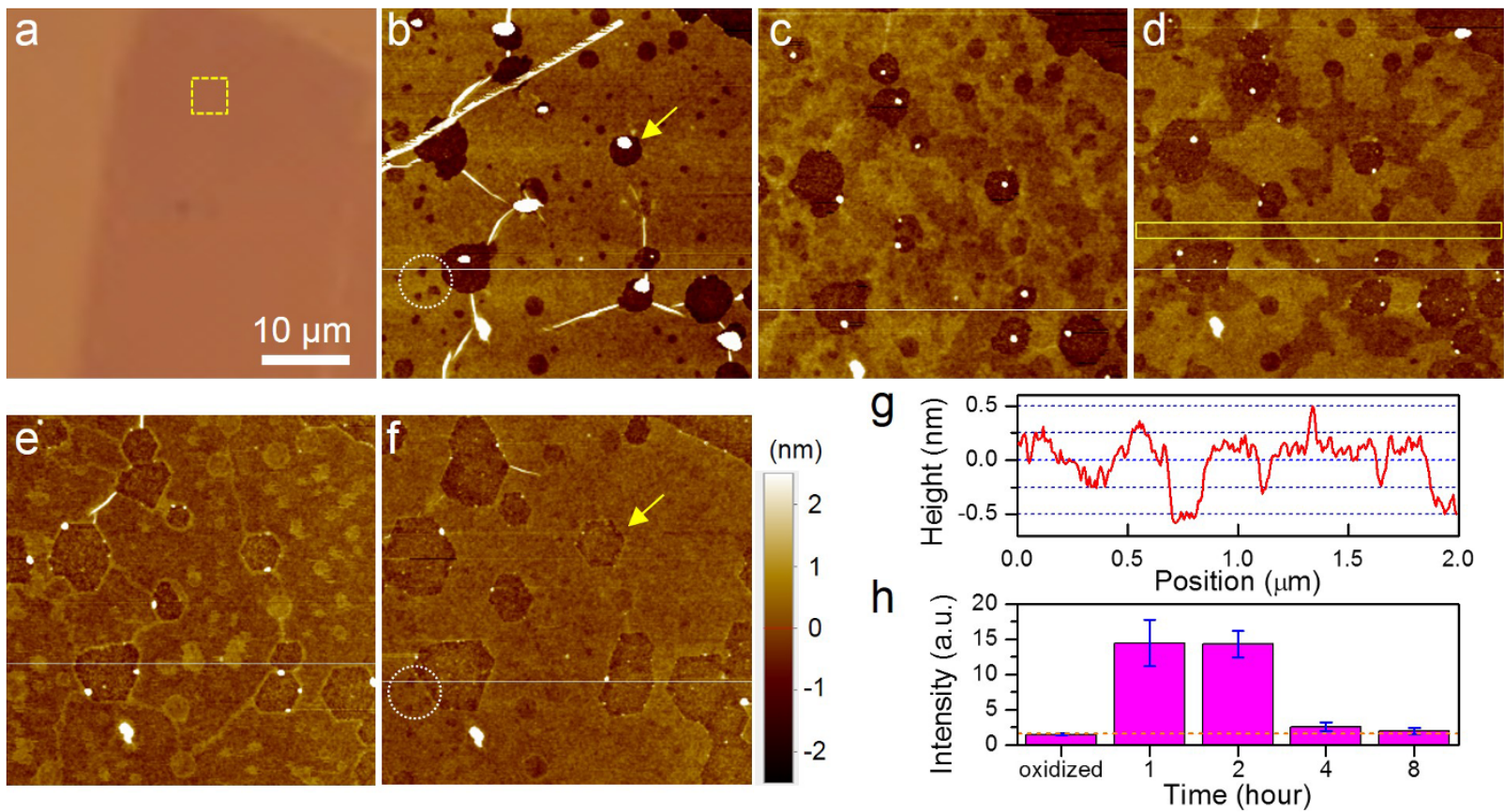

Figure 3 Topographic confirmation of aC layers on 2L graphene. (a) Optical micrograph of pristine 2L graphene. The dashed yellow square marks the position where the AFM images in (b) (f) were obtained. The square was enlarged by a factor of two for visibility. (b) Non-contact AFM height image of the $2 \mathrm{~L}$ graphene obtained after thermal oxidation at $550^{\circ} \mathrm{C}$ for 4 hours, which generated $1 \mathrm{~L}$-deep (in the dotted circle) and $2 \mathrm{~L}$-deep (marked by the yellow arrow) etch pits. (c) (f) AFM image obtained for varying $\tau_{\text {anneal }}:$ (c) 1 , (d) 2 , (e) 4, (f) 8 hours $\left(\mathrm{T}_{\text {anneal }}=700^{\circ} \mathrm{C}\right.$; in Ar gas). The scanned area in (b) $\sim(\mathrm{f})$ is $2 \times 2 \mu \mathrm{m}^{2}$, respectively. Representative height profiles obtained along the white horizontal lines in (b) (f) are given in Supplementary Fig. S5. (g) The height profile averaged over the yellow rectangle in (d). (h) $I\left(D_{a C}\right)$ as a function of $\tau_{\text {anneal }}$. The orange dashed line represents the average $I\left(D_{a C}\right)$ of pristine graphene. 
$550^{\circ} \mathrm{C}$ as shown in Fig. 3b. Then, the sample was annealed in Ar gas at $700^{\circ} \mathrm{C}$ repeatedly for $\tau_{\text {anneal }}=1,2,4$ and 8 hours with the AFM images in Figs. $3 \mathrm{~b} \sim 3 \mathrm{f}$ obtained after each thermal treatment. Most of large pits (marked by the yellow arrow, diameter $>100 \mathrm{~nm}$ ) shown in Fig. $3 b$ turned out to grow into polygonal shapes for $\tau_{\text {anneal }}$ $=8$ hours (Fig. $3 \mathrm{f}$ ) through the carbothermal reduction ${ }^{25}$, indicating that they are $2 \mathrm{~L}$-deep pits ${ }^{7}$ exposing the $\mathrm{SiO}_{2}$ substrate. On the contrary, smaller pits of $1 \mathrm{~L}$ depth ${ }^{7}$ formed on the top graphene layer (encircled by the dotted line in Fig. 3b) remained same in size after 8hour annealing (Fig. 3f) since the carbothermal reduction requires contact between $\mathrm{SiO}_{2}$ and graphene edges ${ }^{25}$. Besides the anisotropic etching, the AFM images also reveal the presence and transformation of thin layers on top of graphene. Whereas the surface of oxidized graphene is flat except the pits (Fig. 3b), the subsequent one-hour annealing led to rather rugged surface (Fig. 3c) and additional treatment generated nicely stepped surface in Fig. $3 \mathrm{~d}$. The height profile averaged the yellow rectangle in Fig. 3d shows that the thickness of the overlayers is $0.5 \sim 0.7 \mathrm{~nm}$ (Fig. $3 \mathrm{~g}$ ). The third round of annealing removed most of the overlayers except those covering the small 1Ldeep pits as shown in Fig. 3e. Finally, annealing for accumulated duration of 8 hours completely cleaned the surface as can be seen Fig. 3f. (See Supplementary Fig. S5 online for detailed height profile change for each of the AFM image.) Figure $3 \mathrm{~h}$ presents $I\left(D_{a C}\right)$ obtained after each thermal treatment. The $\mathrm{D}_{\mathrm{aC}}$ peak was most intense for $\tau_{\text {anneal }}=1$ hour and almost disappeared for $\tau_{\text {anneal }} \geq 4$ hours, showing a good correlation with the topographical details of the overlayers revealed by the AFM images. Comparison of Fig. 2b and Fig. $3 \mathrm{~h}$ leads us to a conclusion that prolonged annealing at $700^{\circ} \mathrm{C}$ is more efficient in removing the aC layers than at $400^{\circ} \mathrm{C}$.

Interestingly, the morphology of the aC overlayers was found to be very different when annealed at $400^{\circ} \mathrm{C}$ in low vacuum. Instead of the planar structures observed in Fig. 3, the graphene annealed for $30 \mathrm{~min}$ is now covered with numerous tiny dots, which disappeared almost completely when annealed for 8 hours (see Supplementary Fig. S6 online). The height distribution of the 8-hour annealed graphene shown in Supplementary Fig. S6 is fitted well by a Gaussian function with FWHM (full width at half maximum) of $350 \mathrm{pm}$, which is essentially equivalent to that of pristine graphene (340 \pm $15 \mathrm{pm}$ ). However, 30-min annealed graphene with the small dots shows an asymmetric distribution with much larger width. Decomposition with two Gaussians led to FWHM's of 390 and $600 \mathrm{pm}$. While the former is due to graphene itself, the latter can be attributed to the amorphous carbon nano-dots. These morphological changes are also consistent with the results obtained by the optical characterization represented by the variation of $\mathrm{I}\left(\mathrm{D}_{\mathrm{aC}}\right)$ in Fig. 2b.

\section{Discussion}

Our study shows that graphene is easily covered by hydrocarbons generated at elevated temperatures from polymers which are often unintentionally provided during the typical sample and device fabrication processes ${ }^{1,23}$. This demands extra caution in interpreting experimental results from graphene samples or devices that have undergone such processes. In particular, the presence of adhesive residues generated during micro-mechanical exfoliation has rarely been taken into account ${ }^{7,26}$, since the residues are typically not in direct contact with graphene flakes of interest. Through this study, however, it has been shown that such residues can adsorb on graphene flakes at elevated temperature. In this regard, it is also to be noted that isolated graphene flakes was contaminated by the polymeric residues dissolved and diffused by common solvents like acetone in a simple dipping process ${ }^{26}$. Some of the hydrocarbons may originate from the ambient air as $\mathrm{Z}$. Li et al. recently showed in airborne contaminants-induced hydrophobicity of graphene ${ }^{27}$.

Furthermore, our study clearly shows that the polymeric adsorbates are not readily removed by brief thermal annealing, which is consistent with the recent observation by transmission electron microscopy (TEM) ${ }^{24}$. As long as non-oxidizing, the nature of ambient gases in the heating chamber was found not to affect the efficiency of the removal greatly in contrast to the common preference for reducing atmosphere including hydrogen ${ }^{9,28}$ rather than noble gases. In fact, the removal efficiency increased as decreasing the pressure inside the chamber from 1 atm to high vacuum, suggesting that gaseous species generated at elevated temperature adsorb on graphene. However, the observed pressure dependence is apparently very modest: a pressure decrease by several orders of magnitude led only to $50 \sim 70 \%$ decrease in $\mathrm{I}\left(\mathrm{D}_{\mathrm{aC}}\right)$. This suggests that graphene is also wetted by some fluidic adsorbates which migrate on the substrates, not necessarily airborne, at high temperature. Alternatively, this may be due to inefficient diffusion of the airborne species from the sample to the vacuum pump. Whereas even chemical species with high molecular mass can be evaporated from the sample located in the heating zone of the quartz tube, they will collide and condense on the less warm inner walls near the ends of the tube which remain near room temperature. The resulting large concentration gradient of the gaseous species along the tube axis will lead to reduced dependence of the efficiency on the base pressure which is measured at the cold end of the tube.

Finally, the current study provides a simple but reliable optical method to determine the amount of amorphous carbon. Although thermal degradation has been employed to remove various adsorbates on graphene samples, it has been not straightforward to confirm each cleanup process since the kinetics of decomposition varies widely from sample to sample according to the nature and amount of the foreign materials. Despite its preciseness ${ }^{24}$, for example, TEM cannot be applied to graphene supported on conventional thick substrates. As shown in the current report, quantification of the amount of $\mathrm{aC}$ with $\mathrm{AFM}$ is also subject to technical difficulties since the morphology of the aC overlayers can be indistinct depending on the specific details of thermal treatments, besides the fact that the method is time-consuming. In contrast, Raman spectroscopy can be carried out quickly without any extra preparation for samples. By simple comparison with a reference spectrum of pristine graphene, the amount of $\mathrm{aC}$ can be precisely determined through $\mathrm{I}\left(\mathrm{D}_{\mathrm{aC}}\right)$ and $\mathrm{I}\left(\mathrm{G}_{\mathrm{aC}}\right)$ at least on a relative scale. Using this analysis, it was demonstrated that aC originating from polymeric residues can be removed in both exfoliated and CVD-grown graphene by extended annealing in vacuum. This should be of practical importance to those who have to ensure surface cleanness of graphene.

In conclusion, we have investigated the Raman spectra of graphene annealed in inert gas or vacuum. In addition to the previously reported spectral shifts of $\mathrm{G}$ and $2 \mathrm{D}$ peaks, very broad additional Raman peaks were found near D, G and 2D frequencies. The new Raman peaks were attributed to amorphous carbon formed on the surface of graphene by carbonization of organic adsorbates originating from coexisting polymeric hydrocarbons in oxygen-free environment.

\section{Methods}

Sample preparation. One group of graphene samples were prepared by the micromechanical exfoliation method ${ }^{1}$ using kish graphite (Covalent Materials Inc.) and $\mathrm{Si}$ substrates topped with $285 \mathrm{~nm}$-thick $\mathrm{SiO}_{2}$ layer. Another group of graphene samples were first grown on $\mathrm{Cu}$ foils by $\mathrm{CVD}$ and transferred onto the $\mathrm{Si} / \mathrm{SiO}_{2}$ substrates followed by chemical etching of the $\mathrm{Cu}$ foils ${ }^{23}$. Thin PMMA support layers were spin-coated on graphene before etching and removed by acetone after transfer ${ }^{23}$.

Raman spectroscopy. The number of layers and structural quality of the samples were characterized by Raman spectroscopy ${ }^{18}$. Raman spectra were obtained with an Ar ion laser operated at $514.5 \mathrm{~nm}$ with average power of $1.5 \mathrm{~mW}$ focused onto a spot of $\sim 0.5 \mu \mathrm{m}$ in diameter by a 40 times objective with numerical aperture of 0.60 . The spectral accuracy was found to be better than $1.0 \mathrm{~cm}^{-1}$ with respect to Raman standards of graphite and Si crystals, whereas the full width at half maximum (FWHM) of the Rayleigh line was $6.0 \mathrm{~cm}^{-1}$ using a spectrograph with focal length of $30 \mathrm{~cm}$ and a grating with 600 grooves $/ \mathrm{mm}^{13}$. To obtain statistical significance, for each set of the experimental conditions, Raman spectra were obtained from $5 \sim$ 
10 spots $(1 \sim 2 \mu \mathrm{m}$ apart $)$ per each of a few graphene flakes larger than $10 \mu \mathrm{m}$ along the long axis.

Thermal treatments. Thermal annealing consisted of linear ramping to a target temperature $\left(\mathrm{T}_{\text {anneal }}\right)$ in $30 \mathrm{~min}$, holding temperature at $\mathrm{T}_{\text {anneal }}$ for a given time ( $\tau_{\text {anneal }}$ ), and cooling down to $\sim 23^{\circ} \mathrm{C}$ for $\sim 3$ hours. To test the effects of gas environment in the quartz tube furnace, annealing was carried out in Ar gas (flow rate of $300 \mathrm{~mL} / \mathrm{min}, \sim 1 \mathrm{~atm})$, forming gas $\left(5 \% \mathrm{H}_{2}\right.$ in $\mathrm{N}_{2}$, flow rate of $300 \mathrm{~mL} / \mathrm{min}$, $\sim 1 \mathrm{~atm}$ ), low vacuum (base pressure of $\sim 2 \times 10^{-3}$ Torr), and high vacuum (base pressure of $\sim 3 \times 10^{-5}$ and $\sim 1 \times 10^{-7}$ Torr). For oxidation, the temperature of samples was varied in the same manner as the thermal annealing, but in a mixed gas atmosphere $\left(\mathrm{O}_{2}: \mathrm{Ar}=350 \mathrm{~mL} / \mathrm{min}: 700 \mathrm{~mL} / \mathrm{min}, \sim 1 \mathrm{~atm}\right)$.

1. Novoselov, K. S. et al. Electric field effect in atomically thin carbon films. Science 306, 666-669 (2004)

2. Dreyer, D. R., Park, S., Bielawski, C. W. \& Ruoff, R. S. The chemistry of graphene oxide. Chem. Soc. Rev. 39, 228-240 (2010).

3. Li, X. S. et al. Large-area synthesis of high-quality and uniform graphene films on copper foils. Science 324, 1312-1314 (2009).

4. Lee, C., Wei, X., Kysar, J. W. \& Hone, J. Measurement of the elastic properties and intrinsic strength of monolayer graphene. Science 321, 385-388 (2008).

5. Ryu, S. et al. Reversible basal plane hydrogenation of graphene. Nano Lett. 8 , 4597-4602 (2008).

6. Elias, D. C. et al. Control of graphene's properties by reversible hydrogenation: Evidence for graphane. Science 323, 610 (2009).

7. Liu, L. et al. Graphene oxidation: Thickness dependent etching and strong chemical doping. Nano Lett. 8, 1965-1970 (2008).

8. Stolyarova, E. et al. High-resolution scanning tunneling microscopy imaging of mesoscopic graphene sheets on an insulating surface. Proc. Natl. Acad. Sci. U.S.A. 104, 9209-9212 (2007)

9. Ishigami, M., Chen, J. H., Cullen, W. G., Fuhrer, M. S. \& Williams, E. D. Atomic structure of graphene on $\mathrm{SiO} 2$. Nano Lett. 7, 1643-1648 (2007).

10. Lee, W. H. et al. Surface-directed molecular assembly of pentacene on monolayer graphene for high-performance organic transistors. J. Am. Chem. Soc. 133, 4447-4454 (2011).

11. Liu, Z. et al. Direct growth of graphene/hexagonal boron nitride stacked layers. Nano Lett. 11, 2032-2037 (2011).

12. Bao, W. et al. Controlled ripple texturing of suspended graphene and ultrathin graphite membranes. Nat. Nanotechnol. 4, 562-566 (2009).

13. Lee, J. E., Ahn, G., Shim, J., Lee, Y. S. \& Ryu, S. Optical separation of mechanical strain from charge doping in graphene. Nat. Commun. 3, 1024 (2012).

14. Ryu, S. et al. Atmospheric oxygen binding and hole doping in deformed graphene on a SiO2 substrate. Nano Lett. 10, 4944-4951 (2010).

15. Cullen, W. G. et al. High-fidelity conformation of graphene to $\mathrm{SiO} 2$ topographic features. Phys. Rev. Lett. 105, 215504 (2010).

16. Yoon, D., Son, Y. W. \& Cheong, H. Negative thermal expansion coefficient of graphene measured by raman spectroscopy. Nano Lett. 11, 3227-3231 (2011).

17. Cheng, Z. G. et al. Toward intrinsic graphene surfaces: A systematic study on thermal annealing and wet-chemical treatment of $\mathrm{SiO} 2$-supported graphene devices. Nano Lett. 11, 767-771 (2011).

18. Ferrari, A. C. et al. Raman spectrum of graphene and graphene layers. Phys. Rev. Lett. 97, 187401/187401-187401/187404 (2006).
19. Basko, D. M., Piscanec, S. \& Ferrari, A. C. Electron-electron interactions and doping dependence of the two-phonon raman intensity in graphene. Phys. Rev. B 80, 165413 (2009).

20. Ferrari, A. C. \& Robertson, J. Interpretation of raman spectra of disordered and amorphous carbon. Phys. Rev. B 61, 14095-14107 (2000).

21. Ferrari, A. C. Raman spectroscopy of graphene and graphite: Disorder, electron phonon coupling, doping and nonadiabatic effects. Solid State Commun. 143, 47-57 (2007).

22. Kinoshita, K. Carbon-electrochemical and physicochemical properties. 56 (John Wiley \& Sons, 1988)

23. Bae, S. et al. Roll-to-roll production of 30-inch graphene films for transparent electrodes. Nat. Nanotechnol. 5, 574-578 (2010).

24. Lin, Y. C. et al. Graphene annealing: How clean can it be? Nano Lett. 12, 414-419 (2012).

25. Nemes-Incze, P., Magda, G., Kamaras, K. \& Biro, L. P. Crystallographically selective nanopatterning of graphene on SiO2. Nano Res. 3, 110-116 (2010).

26. Bruna, M. \& Borini, S. Assessment of graphene quality by quantitative optical contrast analysis. J. Phys. D Appl. Phys. 42, 175307 (2009)

27. $\mathrm{Li}, \mathrm{Z}$. et al. Effect of airborne contaminants on the wettability of supported graphene and graphite. Nat. Mater., published online (2013), http://dx.doi.org/ 2010.1038/nmat3709.

28. Ping, J. L. \& Fuhrer, M. S. Layer number and stacking sequence imaging of fewlayer graphene by transmission electron microscopy. Nano Lett. 12, 4635-4641 (2012).

\section{Acknowledgements}

This work was supported by the National Research Foundation of Korea (No. 2012-053500, 2012-043136, 2012-0003059). The authors thank Hyeongkeun Kim and Sugang Bae for the access to high vacuum furnaces and Byung Hee Hong for providing CVD-grown graphene samples. The authors are also grateful to Chaiwon Kwon for providing carbon black powder and Taeg Yeoung Ko for helpful comments.

\section{Author contributions}

S.R. proposed and supervised the entire project. J.H., M.K.P., E.J.L., D.E.L. and D.S.H. performed the experiments. J.H., M.K.P., E.J.L. and S.R. analysed the data. S.R. wrote the manuscript and all the authors participated in discussions of the research.

\section{Additional information}

Supplementary information accompanies this paper at http://www.nature.com/ scientificreports

Competing financial interests: The authors declare no competing financial interests.

How to cite this article: Hong, J. et al. Origin of New Broad Raman D and G Peaks in Annealed Graphene. Sci. Rep. 3, 2700; DOI:10.1038/srep02700 (2013).

(c) (i) $\Theta$ This work is licensed under a Creative Commons AttributionBy NG No NonCommercial-NoDerivs 3.0 Unported license. To view a copy of this license, visit http://creativecommons.org/licenses/by-nc-nd/3.0 